\title{
Lessons of the month 2: Ocular manifestations and complications of hepatitis $\mathrm{C}$ infection
}

\author{
Authors: Sarah LW Fang, ${ }^{\mathrm{A}}$ Livia Teo, ${ }^{\mathrm{B}}$ Cheng Yi Loo, ${ }^{\mathrm{C}}$ James $\mathrm{W} \mathrm{Li}^{\mathrm{D}}$ and Anindita Santosa ${ }^{\mathrm{E}}$
}

We present a case of a man with hepatitis $C$ with acute visual loss in his left eye over a 3-day period. Initial evaluation suggested a possible idiopathic orbital inflammatory syndrome as underlying cause for his symptoms. Initial response to systemic glucocorticoids and antiviral treatment were encouraging with improvement in the patient's visual acuity. After a period of loss to follow up and discontinuation of treatment, the patient re-presented with visual loss in his left eye. Re-evaluation confirmed a diagnosis of marginal zone B-cell lymphoma. This case illustrates the importance of considering the various ocular manifestations of hepatitis $C$.

KEYWORDS: ocular lymphoma, hepatitis C, orbital inflammatory syndrome

DOI: $10.7861 /$ clinmed.2021-0320

\section{Case presentation}

We present a case of a 47-year-old man who was admitted for acute blurring of vision of his left eye over 3 days associated with nausea and vomiting. Prior to this, he reported a 1-month history of bilateral headache without any focal neurological symptoms. He was otherwise well with a history of previous acute urethritis, gastritis and well-controlled type 2 diabetes mellitus. He had no long-term medications. On examination, there was proptosis of the left eye. The left pupillary light reflex was sluggish, associated with a relative afferent pupillary defect (RAPD) and loss of colour perception. Left ocular movements were restricted in all directions.

The patient underwent computed tomography (CT), which revealed a left orbital apex mass with infiltration of the left medial and inferior rectus with encasement of the left optic nerve. He was also incidentally noted to have a cirrhotic liver. Magnetic resonance imaging (MRI) of the orbit showed a left orbital homogeneously enhancing mass inseparable from the left medial and inferior recti muscles (Fig 1).

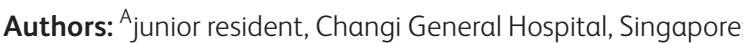
${ }^{B}$ senior consultant in ophthalmology, Singapore National Eye Centre, Singapore; ' medical officer, Singapore National Eye Centre, Singapore; ${ }^{\mathrm{D}}$ consultant in gastroenterology and hepatology, Changi General Hospital, Singapore; ${ }^{\mathrm{E}}$ Consultant in rheumatology, Changi General Hospital, Singapore
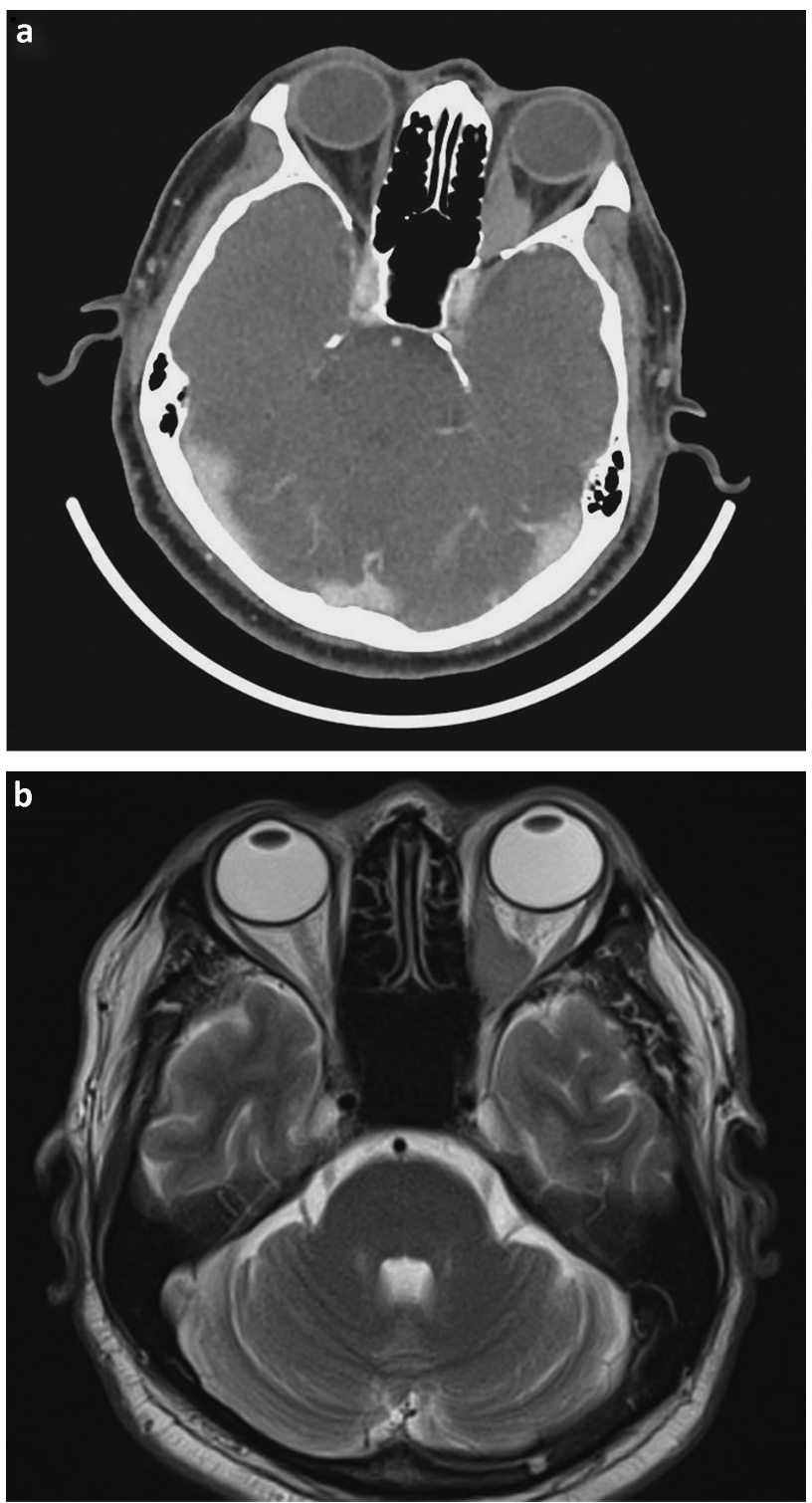

Fig 1. a) Computed tomography of the brain showing left orbital mass with infiltration of the left medial rectus. b) Magnetic resonance of the brain/orbit T2-weighted image showing the left orbital homogeneouslyenhancing mass with compression of the optic nerve. 
Fig 2. After treatment with high-dose oral steroids and antiviral therapy showing near-resolution of restriction in left extra-ocular movements.
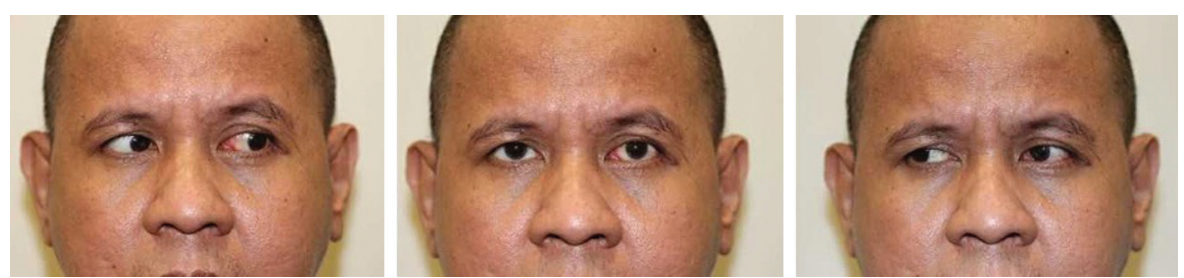

Hepatitis $\mathrm{C}$ virus (HCV) serology was positive with a viral load of $1.95 \times 10^{5} \mathrm{IU} / \mathrm{mL}$. The liver panel was normal except for a borderline raised gamma-glutamyltransferase. His autoimmune screen was negative.

He underwent a left orbitotomy with biopsy of the orbital mass. Histopathological examination revealed non-specific inflammatory infiltrates and microbiology tests were negative. Hence, a working diagnosis of orbital inflammatory syndrome (OIS) secondary to HCV was made.

\section{Treatment, progress and outcome}

The patient was started on systemic glucocorticoids soon after the orbital biopsy was performed (intravenous methylprednisolone $250 \mathrm{mg}$ every 6 hours). He was also started on treatment for HCV (sofosbuvir/velpatasvir 1 tablet once daily and ribavirin $600 \mathrm{mg}$ once daily). He was discharged with a high dose of oral steroids. During follow-up appointments, he was found to have drastic improvements in visual acuity and extra-ocular movements. There were undetectable viral titres after treatment with sofosbuvir/ velpatasvir after 12 weeks (Fig 2).

Despite good initial response to HCV treatment and systemic glucocorticoids, the patient became non-adherent to therapy and follow-up after 2 weeks. He re-presented with recurrence of visual loss in his left eye after 2 months and new onset left facial and upper limb numbness. The new neurological symptoms did not appear to be in keeping with the diagnosis of OIS. Hence, the decision was made to re-evaluate him for other possible causes for his condition.

Repeat imaging of the brain and cervical spinal cord and analysis of the cerebrospinal fluid (including flow cytometry for lymphoma) were unremarkable. A repeat biopsy of the retro-orbital mass via trans-sphenoidal route was performed and the histology was consistent with extra-nodal marginal zone B-cell lymphoma involving the skeletal muscles. He was referred to a haematologist and management of extra-nodal lymphoma was initiated.

\section{Discussion}

Infection with $\mathrm{HCV}$ is rare in Singapore, with an estimated prevalence rate of about $0.2 \%$. HCV infection is associated with hepatic and extrahepatic sequalae which are thought to be a result of immune-dysregulation caused by the virus. Up to $74 \%$ of individuals with chronic HCV infection develop extrahepatic manifestations.

\section{Hepatitis C and lymphoma; what is the link?}

Lymphoproliferative disorders are the most common extrahepatic manifestations of HCV. ${ }^{1}$ Current epidemiological studies have demonstrated a link between HCV infections and the development of non-Hodgkin lymphoma (NHL). HCV lymphomagenesis is postulated to involve various mechanisms including chronic antigenic stimulation in the early stages and direct transformation by viral proteins in later stages. ${ }^{2}$ Studies have demonstrated efficacy in lymphoma regression in response to anti-HCV therapy which further supports this hypothesis. ${ }^{3}$

\section{What is the role of anti-viral therapy in HCV-associated lymphoma?}

The role of antiviral therapy in HCV-associated lymphoma is well established. In recent years, literature has established the benefits of the first-line antiviral therapy with interferon in low grade NHLs. Prospective studies as well as systematic meta-analyses in HCV-associated low-grade B-NHLs have shown a global response rate to antiviral therapy of $70 \%$, suggesting a strong role of anti-viral treatment in HCV-associated lymphomas. ${ }^{4,5}$ Sustained viral response (SVR) after antiviral therapy has also shown to be associated with a better overall survival in HCV-related lymphoma. ${ }^{4}$

\section{What are the ocular manifestations of hepatitis C?}

To date, there are no pathognomonic manifestations of HCV infection in the eye, though some ocular syndromes have been reported in case series and reports. The common ocular manifestations in HCV include ischaemic retinopathy (due to HCVinduced vasculitis) and keratoconjunctivitis sicca. ${ }^{6}$

The overall incidence of ocular lymphoma in association with $\mathrm{HCV}$ is still low as only a few studies have explored the association of HCV infection and ocular adnexal lymphomas (ie conjunctiva, lacrimal gland and orbital soft tissue), although the ocular region is one of the most common sites of extra-nodal lymphomas., ${ }^{7,8}$

The incidence of orbital inflammatory syndrome is also rare in patients with HCV and is less prevalent than other ocular manifestations. They typical respond rapidly to high-dose steroids and anti-viral therapy. The diagnosis of OIS is made after the exclusion of other identifiable local and systemic causes of orbital inflammation.

\section{Conclusion}

In this case, the initial biopsy suggested an idiopathic inflammatory cause. A high index of suspicion prompted the clinicians involved to obtain a second biopsy which eventually clinched the diagnosis of marginal zone B-cell lymphoma. We suggest that close follow-up of ocular symptoms and interval imaging is warranted in patients with hepatitis $C$ and evidence of ocular involvement. 


\section{Key points}

> Many individuals with chronic HCV infection develop extrahepatic manifestations, therefore vigilance is required in recognising such complications when monitoring patients with hepatitis $C$.

> Although HCV-associated ocular lymphomas are rare, clinicians should have an increased index of suspicion in HCV infections with ocular involvement. Close monitoring of symptoms and serial imaging are warranted in such cases.

> There is a strong role for antiviral therapy in HCV-associated lymphomas and it may improve overall survival.

\section{References}

1 Cacoub P, Gragnani L, Comarmond C, Zignego AL. Extrahepatic manifestations of chronic hepatitis C virus infection. Dig Liver Dis 2014;46(Suppl 5):S165-73.

2 Armand M, Besson C, Hermine O, Davi F. Hepatitis C virus Associated marginal zone lymphoma. Best Pract Res Clin Haematol 2017:30:41-9.

3 Persico M, Aglitti A, Caruso R et al. Efficacy and safety of new direct antiviral agents in hepatitis $C$ virus-infected patients with diffuse large B-cell non-Hodgkin's lymphoma. Hepatology 2018;67:48-55.

4 Couronné L, Bachy E, Roulland S et al. From hepatitis $C$ virus infection to B-cell lymphoma. Ann Oncol 2018;29:92-100.

5 Peveling-Oberhag J, Arcaini L, Bankov K, Zeuzem S, Herrmann E. The anti-lymphoma activity of antiviral therapy in HCV-associated B-cell non-Hodgkin lymphomas: a meta-analysis. J Viral Hepat 2016;23:536-44.

6 Zegans ME, Anninger W, Chapman C, Gordon SR. Ocular manifestations of hepatitis C virus infection. Curr Opin Ophthalmol 2002:13:423-7.

7 Arnaud P, Escande MC, Lecuit M et al. Hepatitis C virus infection and MALT-type ocular adnexal lymphoma. Ann Oncol 2007:18:400-3.

8 Strianese D, Tranfa F, Finelli M et al. Hepatitis C virus infection in ocular adnexal lymphomas. Arch Ophthalmol 2010;128:1295-9.

Address for correspondence: Dr Sarah LW Fang, Changi General Hospital, 2 Simei Street 3, Singapore 529889. Email: sarah.fang@mohh.com.sg 